\title{
Pitfalls in quantitative myocardial PET perfusion I: Myocardial partial volume correction
}

K. Lance Gould, MD, ${ }^{\mathrm{a}, \mathrm{g}, \mathrm{h}}$ Linh Bui, MD, ${ }^{\mathrm{b}, \mathrm{h}}$ Danai Kitkungvan, MD, ${ }^{\mathrm{b}, \mathrm{h}}$ Tinsu Pan, PhD, ${ }^{c, h}$ Amanda E. Roby, PET, CNMT, RT(N), ${ }^{\text {d,h }}$ Tung T. Nguyen, BS, ${ }^{\mathrm{e}, \mathrm{h}}$ and

Nils P. Johnson, MD, $M S^{f, h}$

a Martin Bucksbaum Distinguished University Chair, Weatherhead P.E.T. Center for Preventing and Reversing Atherosclerosis, McGovern Medical School, University of Texas Health Science Center, Houston, TX

b Division of Cardiology, McGovern Medical School, UT Health - Houston, Houston, TX

c Imaging Physics Department, MD Anderson Cancer, University of Texas, Houston, TX

d Weatherhead PET Center, McGovern Medical School, Houston, TX

e Programming and Data Management, Weatherhead P.E.T. Center, McGovern Medical School, University of Texas, Houston, TX

f Weatherhead Distinguished Chair of Heart Disease, Division of Cardiology, McGovern Medical School, Houston, TX

g Weatherhead PET Center For Preventing and Reversing Atherosclerosis, McGovern Medical School, University of Texas Health Science Center at Houston, Houston, TX

h Weatherhead PET Center For Preventing and Reversing Atherosclerosis, Division of Cardiology, Department of Medicine, McGovern Medial Medical School, University of Texas, and Memorial Hermann Hospital, Houston, TX

Received Jun 15, 2019; accepted Oct 24, 2019

doi: $10.1007 / \mathrm{s} 12350-020-02073-9$

Background. PET quantitative myocardial perfusion requires correction for partial volume loss due to one-dimensional $\mathrm{LV}$ wall thickness smaller than scanner resolution.

Methods. We aimed to assess accuracy of risk stratification for death, MI, or revascularization after PET using partial volume corrections derived from two-dimensional ACR and three-dimensional NEMA phantoms for 3987 diagnostic rest-stress perfusion PETs and 187 MACE events. NEMA, ACR, and Tree phantoms were imaged with Rb-82 or F-18 for sizedependent partial volume loss. Perfusion and Coronary Flow Capacity were recalculated using different ACR- and NEMA-derived partial volume corrections compared by KolmogorovSmirnov statistics to standard perfusion metrics with established correlations with MACE.

Electronic supplementary material The online version of this article (https://doi.org/10.1007/s12350-020-02073-9) contains supplementary material, which is available to authorized users.

The authors of this article have provided a PowerPoint file, available for download at SpringerLink, which summarises the contents of the paper and is free for re-use at meetings and presentations. Search for the article DOI on SpringerLink.com.

The authors have also provided an audio summary of the article, which is available to download as ESM, or to listen to via the JNC/ASNC Podcast.

Funding Research supported by internal funds of the Weatherhead PET Center: NPJ received institutional licensing and consulting agreement with Boston Scientific for the smart minimum FFR algorithm; received significant institutional research support from St. Jude Medical (CONTRAST, NCT02184117) and Philips Volcano Corporation (DEFINE-FLOW, NCT02328820) for studies using intracoronary pressure and flow sensors; and has a patent pending on diagnostic methods for quantifying aortic stenosis and TAVI physiology. KLG receives internal funding from the Weatherhead PET Center for Preventing and Reversing Atherosclerosis and is the $510(\mathrm{k})$ applicant for FDA approved HeartSee K171303 PET software. To avoid any conflict of interest, KLG has assigned any royalties to UT for research or student scholarships, has no consulting, speakers, or board agreements, and receives no funding from PET related companies.

Reprint requests: K. Lance Gould, MD, Weatherhead PET Center For Preventing and Reversing Atherosclerosis, McGovern Medical School, University of Texas Health Science Center at Houston, 6431 Fannin St., Room MSB 4.256, Houston, TX 77030; k.lance.gould@uth.tmc.edu 1071-3581/\$34.00

Copyright (c) 2020 The Author(s) 
Results. Partial volume corrections based on two-dimensional ACR rods (two equal radii) and three-dimensional NEMA spheres (three equal radii) over estimate partial volume corrections, quantitative perfusion, and Coronary Flow Capacity by $50 \%$ to $150 \%$ over perfusion metrics with one-dimensional partial volume correction, thereby substantially impairing correct risk stratification.

Conclusions. ACR (2-dimensional) and NEMA (3-dimensional) phantoms overestimate partial volume corrections for 1-dimensional $L V$ wall thickness and myocardial perfusion that are corrected with a simple equation that correlates with MACE for optimal risk stratification applicable to most PET-CT scanners for quantifying myocardial perfusion. (J Nucl Cardiol 2020;27:386-96.)

Key Words: Cardiac positron emission tomography $($ PET) $・$ quantitative myocardial perfusion - coronary flow reserve $\cdot$ partial volume correction - ACR or NEMA PET phantoms

\begin{tabular}{|ll|}
\hline Abbreviations & \\
ACR & American College of Radiology \\
NEMA & National Electrical Manufactures \\
& Association \\
CAD & Coronary artery disease \\
CFC & Coronary flow capacity \\
KS & Kolmogorov-Smirnov statistic \\
LV & Left ventricule \\
MACE & Major adverse cardiovascular events \\
& (here death, MI, revascularization) \\
PET & Positron emission tomography \\
PV & Partial volume \\
ROI & Region of interest \\
\hline
\end{tabular}

\section{INTRODUCTION}

Quantitative myocardial perfusion by positron emission tomography (PET) requires correction for partial volume (PV) loss due to left ventricular (LV) wall thickness being smaller than scanner resolution. Currently, myocardial perfusion by PET is calculated by either of two different perfusion models accounting for PV loss. One model uses time activity curves from arterial and myocardial regions of interest (ROI) on serial, short-duration images fit to a compartmental perfusion model to solve for unknown reconstruction parameters, one of which is PV correction and perfusion; these PV values are not listed explicitly or published but "buried" within flow model equations. ${ }^{1,2}$ Consequently, this model is less suited for studying effects of PV corrections on perfusion values and resulting patient management decisions or clinical outcomes addressed here.

Alternatively, a validated "simple" or "retention" perfusion model uses a fixed arterial phase image (2 minutes for Rb-82) followed by a fixed myocardial phase acquisition (5 minutes for $\mathrm{Rb}-82)^{3-13}$ validated experimentally ${ }^{3}$ as equivalent to fitting time activity curves of a compartmental model for quantitative perfusion and applied clinically (2-16). Equations for the "simple" model use a fixed PV correction determined by imaging phantoms with different size targets from which activity loss is determined as a fraction of known activity.

The one dimension of LV wall thickness is less than PET scanner resolution, whereas LV circumferential and longitudinal dimensions are substantially larger than scanner resolution. ${ }^{4}$ Therefore, the one dimension of LV wall thickness varying through systole and diastole determines the heart rate dependent partial volume loss for quantitative myocardial perfusion by $\mathrm{PET}^{4}$ not accounted for by heart models with or without defects. Consequently, we tested the following hypothesis using the retention perfusion model: (A) The two-dimensional American College of Radiology (ACR) phantom rods (two equal radii) or three-dimensional National Electrical Manufacturers Association (NEMA) phantom spheres (three equal radii) to determine myocardial PV loss and corrections overestimate values compared to a one-dimensional reference phantom. (B) Compared to validated low perfusion thresholds associated with ischemia using PV correction derived from one-dimensional limiting wall thickness of the $\mathrm{LV}^{3-13}$ overcorrection for PV loss based on ACR or NEMA phantoms results in erroneously high perfusion with consequent impaired risk stratification for MACE, death, or their reduction after revascularization.

\section{METHODS}

\section{Background and Rationale}

In cardiac PET, point spread function and loss of peak activity recovery are due to several factors as previously detailed ${ }^{4}$ : (i) Limited scanner resolution of $10 \mathrm{~mm}$ to $20 \mathrm{~mm}$ full width at half maximum (FWHM); (ii) Positron range; and (iii) Reconstruction parameters and filters. 


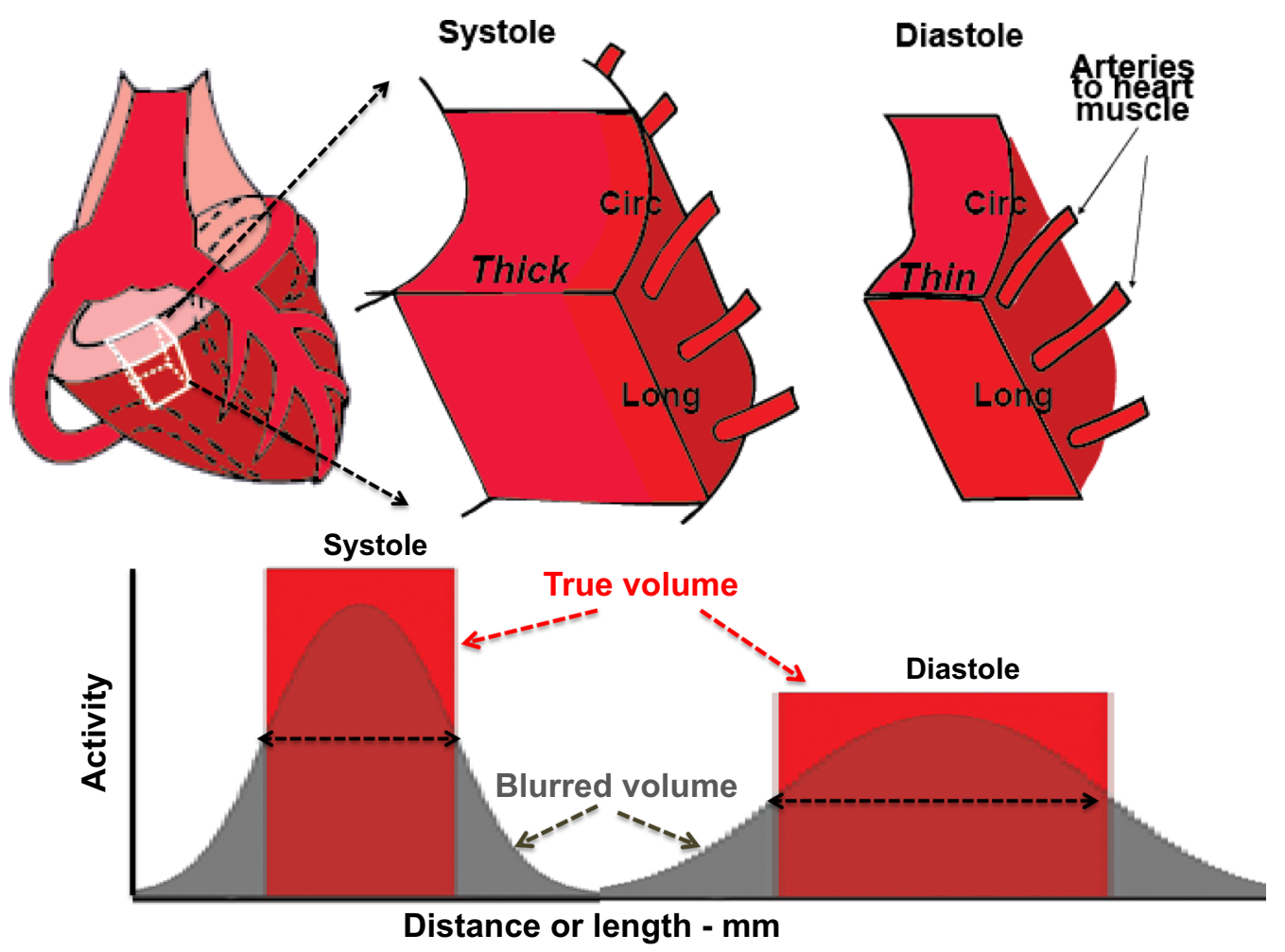

Figure 1. Schema of LV wall with partial volume activity loss due to LV wall thickness during cardiac cycle averaging approximately $15 \mathrm{~mm}$, whereas circumferential (Circ) and longitudinal dimensions (Long) are larger than scanner resolution thereby not contributing to partial volume loss.

The left ventricle (LV) is a large, tapered cylinder of varying wall thickness during systole and diastole (Figure 1). Circumferential and longitudinal dimensions of LV exceed scanner resolution throughout the heart cycle, thereby engendering negligible PV loss for these dimensions except at the apex. Observed myocardial activity in diastole of $20 \%$ to $50 \%$ less than in systole is due to diastolic wall thickness less than scanner resolution; thus, myocardial PV loss is due to the single dimension of $\mathrm{LV}$ wall thickness since circumferential and long axis dimensions are larger than scanner resolution.

PV corrections for cardiac PET are complex for several reasons not accounted for by static, standard phantom measurements and calculations. ${ }^{4}$ The first is failure to account for the unique spatial dimensions of small LV wall thickness with large circumferential and longitudinal dimensions compared to scanner resolution. Secondly, LV wall thickness dynamically changes from systole to diastole with corresponding variable PV loss depending on heart rate. ${ }^{4}$ Accordingly, an approximate average $\mathrm{LV}$ wall thickness of $15 \mathrm{~mm}$ during whole heart cycles as previously reported ${ }^{4}$ was used for estimating one-dimensional PV corrections in patient studies.

This partial volume correction factor is inserted into the equation for calculating $\mathrm{cc} / \mathrm{min} / \mathrm{g}$ for each radial pixel as previously reported ${ }^{3-13}$ and in the Online Resource-1 that (i) is highly reproducible $\pm 10 \%$ on test/retest measurement in the same patient within minutes ${ }^{5}$ (ii) correlates with stress induced angina or ST depression ${ }^{4-13}$, and (iii) predicts high risk of death or myocardial infarction ${ }^{6,7}$ that is significantly reduced by revascularization. ${ }^{7}$ In order to show their clinical importance, we address the effects on quantitative perfusion and MACE of various PV corrections derived from the one-dimensional tree phantom branches, the two-dimensional ACR rods, and the three-dimensional NEMA spheres.

\section{Phantom Characteristics}

Three phantoms listed below and shown in Figure 2 were filled with approximately $0.37 \mathrm{MBq} / \mathrm{mL}(10 \mu \mathrm{Ci} /$ $\mathrm{mL}$ ) of R-82 or F-18 that approximates the myocardial 

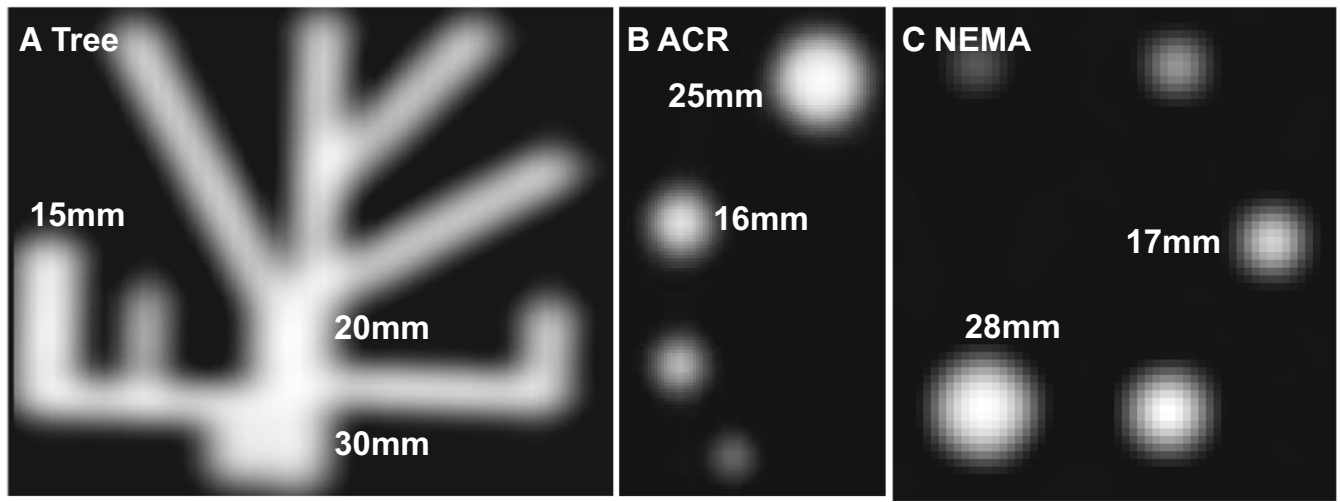

Figure 2. Tomographic images of three phantoms (A) One-dimensional tree phantom with branch widths $<20 \mathrm{~mm}$ and other dimensions $>20 \mathrm{~mm}$. (B) Two-dimensional ACR phantom rods with two equal radii some of which are $<20 \mathrm{~mm}$ and rod length $>20 \mathrm{~mm}$. (C) Three-dimensional NEMA phantom spheres with three equal radii some of which are $<20 \mathrm{~mm}$.

activity observed in our laboratory and imaged by our standard clinical acquisition protocol. ${ }^{3-13}$

NEMA is comprised of 5 spheres of activity each of which is limited in its three dimensions to equal radii of $10 \mathrm{~mm}, 13 \mathrm{~mm}, 17 \mathrm{~mm}, 22 \mathrm{~mm}$, and $28 \mathrm{~mm}$. Therefore, it tests PV loss for a three-dimensional target.

$A C R$ is comprised of 4 rods of activity each of which is limited in its two equal radii of $8 \mathrm{~mm}, 12 \mathrm{~mm}$, $16 \mathrm{~mm}$, and $25 \mathrm{~mm}$ and larger rod length than scanner resolution. Therefore, it tests PV loss for a two-dimensional target.

Tree phantom developed at the University of Texas has rectangular long and deep dimension $>20 \mathrm{~mm}$ with branch width being the only limited dimension of $5 \mathrm{~mm}$, $10 \mathrm{~mm}, 15 \mathrm{~mm}, 20 \mathrm{~mm}$, and $30 \mathrm{~mm}$. It, therefore, tests PV loss for a one-dimensional target.

\section{Generalized Equation for Cardiac Partial Volume Corrections}

Since our Tree phantom is not widely available, we hypothesized that ACR and NEMA phantoms could provide an acceptable approximation of the one-dimensional PV correction using a simple correction (Eq. 1) that accounts for scanner resolution, radionuclide range, and one, two, or three target dimensions less than $20 \mathrm{~mm}$ :

$$
\mathrm{PV}_{\text {observ }}=(R x \text { PV1D })^{n}
$$

where $\mathrm{PV}_{\text {observ }}$ is measured peak activity recovery of either $\mathrm{Rb}-82$ or F-18 expressed as a relative ratio of the $16 \mathrm{~mm} / 25 \mathrm{~mm}$ ACR rods or the $17 \mathrm{~mm} / 28 \mathrm{~mm}$ NEMA spheres. Because the cumulative impact of reconstruction algorithms and smoothing filters affects both narrow $(16-17 \mathrm{~mm})$ and wide $(25-28 \mathrm{~mm})$ targets, it largely cancels out when computing the observed PV ratio. $R$ is the activity recovery loss due to $\mathrm{Rb}-82$ positron range relative to F-18. PV1D equals observed activity recovery as the relative activity ratio of the $15 \mathrm{~mm} / 20 \mathrm{~mm}$ one-dimensional Tree phantom width for either Rb-82 or $\mathrm{F}-18$, and $\mathrm{n}$ is the number of dimensions of each phantom ( 1 for the Tree, 2 for ACR, and 3 for NEMA).

The reconstruction parameters for every cardiac PET scanner should optimize maximal activity recovery with mild smoothing to reduce statistical noise to acceptable images for clinical interpretation that varies with each facility. At these optimized fixed reconstruction parameters, the one-dimensional PV correction for cardiac PET using Rb-82 or F-18 is derived as the relative activity ratio of the $16 \mathrm{~mm} / 25 \mathrm{~mm}$ ACR rods or of the $17 \mathrm{~mm} / 28 \mathrm{~mm}$ NEMA spheres with the above equation rearranged as follows (Eq. 2):

$$
\mathrm{PV} 1 \mathrm{D}=\left(\mathrm{PV}_{\text {observ }} / R^{n}\right)^{1 / n}={ }^{n} \sqrt{ } \mathrm{PV}_{\text {observ }} / R
$$

namely a square root for ACR and cube root for NEMA.

Partial volume correction derived for a phantom is based on "peak activity" recovered for dimensions smaller than scanner resolution. This "peak" partial volume correction must be applied to peak myocardial activity across the LV wall rather than average activity across the wall in order to maintain the principle of preserved area under the activity curve of the point spread function (as the peak of the activity curve decreases, the activity curve widens reflecting increased FWHM). If average activity across the LV wall is used, the partial volume correction needs to be determined by the average activity across the rods of the ACR or spheres of the NEMA phantoms where both are highly variable due tracking borders at statistically poor low counts. 


\section{PET Imaging}

As described previously, ${ }^{3-13}$ rest-stress myocardial perfusion PET was performed using a Discovery ST PET with 16-slice CT scanner (GE Healthcare, Waukesha, Wisconsin) in 2-dimensional mode with extended septa and reconstruction parameters for theoretical inplane resolution of $5.9 \mathrm{~mm}$ full width at half maximum (FWHM) as defined by NEMA standards with pixel size of $3.27 \times 3.27 \mathrm{~mm}$. For patients and all phantoms, images were reconstructed using filtered back projection for $x-y$ plane with a Butterworth filter for the $z$-axis having a cutoff of 0.52 , roll-off of 10 producing uniform comparable activity profiles on short and long axis views of a $20 \mathrm{~cm}$ uniformity phantom.

We used standard dipyridamole, adenosine, or regadenoson stress and $1100-1850 \mathrm{mBq} \quad(30$ to $50 \mathrm{mCi}$ ) of generator-produced Rb-82 (Bracco Diagnostics, Princeton, New Jersey) with low-dose CT optimized attenuation co-registration. ${ }^{4-13}$

Absolute myocardial perfusion was quantified by HeartSee software (FDA approved 510(k) K171303) (-13 $^{4-13}$ using arterial inputs personalized for each PET from among five aortic and left atrium locations ${ }^{4-13}$ yielding $\pm 10 \%$ test-retest precision within minutes in the same patient. ${ }^{5}$ Regional rest and stress flow $(\mathrm{cc} / \mathrm{min} / \mathrm{g})$ and CFR as stress/rest ratio were determined for each of 1344 pixels in the LV. CFC integrates regional pixel values of stress $\mathrm{cc} / \mathrm{min} / \mathrm{g}$ and CFR into 5 color ranges from red (normal, healthy volunteers) to blue (severely reduced with angina or ST changes during stress) as previously reported ${ }^{4-13}$ and detailed in the Online Resource figure.

Subjects undergoing diagnostic PET for quantitative myocardial perfusion since mid 2007 were analyzed as previously reported. ${ }^{4-13}$ All subjects were followed for MACE including all-cause death, non-fatal myocardial infarction, and revascularization after the PET. ${ }^{6,7}$ All subjects signed written informed consent approved by the University of Texas Committee for the Protection of Human Subjects. Quantitative rest/stress PET perfusion and following up for events after PET were obtained from 3987 cases of which 3800 had no MACE and 187 had MACE.

In the absence of an absolute "gold standard" for myocardial activity or perfusion, we used as a "reference standard" the MACE outcomes (death, MI, or revascularization) with and without different PV corrections to demonstrate their importance on clinical management. For 3987 PETs, rest perfusion, stress perfusion, and Coronary Flow Capacity (CFC) per pixel were recalculated for each PET using PV corrections of Table 1 for the ACR $16 \mathrm{~mm}$ two-dimensional rods and the NEMA $17 \mathrm{~mm}$ three-dimensional spheres. Cases were divided into 187 PETs followed by MACE after PET and 3800 without MACE in order to demonstrate the impact of $\mathrm{PV}$ corrections changing perfusion and $\mathrm{CFC}$ sufficiently to impact risk stratification for known MACE.

\section{Statistical Analysis}

Mean \pm standard deviations are reported for continuous variables, number with percent for categorical variables, and mean with one standard deviation for continuous variables with skewed distribution. We utilized paired or unpaired $t$ tests to compare continuous variables and Chi-square or Fisher's exact test to compare categorical data. Kolmogorov-Smirnov (KS) tests compared histogram distributions between groups in color-coded ranges of relative regional uptake images and regional CFC distribution of the left ventricle. ${ }^{5}$

\section{RESULTS}

In Figure 2, spreading effects and PV loss cumulatively from limited resolution, positron range, and reconstruction parameters-filters increase with each additional dimension of target activity less than $20 \mathrm{~mm}$. Therefore, peak recovered activity decreases with each additional target dimension less than $20 \mathrm{~mm}$ since the total cumulative activity under one-, two- or threedimensional area, or volume plots of relative activity has

Table 1. PV loss for 2D GE DST PET-CT with F-18 and Rb-82 in Tree, ACR, NEMA phantoms

\begin{tabular}{lccc}
\hline Phantom & $\begin{array}{c}\text { Tree one dimension } \\
\mathbf{1 5 m m}\end{array}$ & $\begin{array}{c}\text { ACR two dimensions } \\
\mathbf{1 6 \mathbf { m m }}\end{array}$ & $\begin{array}{c}\text { NEMA } \mathbf{3} \text { dimensions } \\
\mathbf{1 7 m m}\end{array}$ \\
\hline F-18 PV loss & 0.94 & 0.85 & 0.72 \\
Rb-82 PV & 0.90 & 0.73 & 0.59 \\
loss & & &
\end{tabular}


to remain constant for fixed activity in the phantom target as also illustrated in Figure $1 .{ }^{4}$

Table 1 shows for F-18 and Rb-82, the progressive PV loss of activity from the one-dimensional tree phantom to two-dimensional ACR phantom to threedimensional NEMA phantom. In addition, PV loss for the $15 \mathrm{~mm}$ one-dimensional Tree phantom and the $16 \mathrm{~mm}$ two-dimensional ACR rods are comparable for Rb-82 and F-18. However, for each target size within the ACR and NEMA phantoms, the PV loss for Rb-82 is greater than for F-18 due to greater positron range.

Figure 3 plots relative activity recovery for $\mathrm{Rb}-82$ (A) and F-18 (B) for approximately comparable size targets of the one-dimensional Tree branch, two-dimensional ACR rod diameters, and three-dimensional NEMA sphere diameters, respectively, as follows in corresponding order of Tree, ACR, NEMA: 10-12$10 \mathrm{~mm}, 15-16-15 \mathrm{~mm}$, and 20-25-30 mm. The largest sizes $>20 \mathrm{~mm}$ show equal activity recovery as the denominator reference for the smaller dimensions for which activity is expressed as a relative ratio to the $20 \mathrm{~mm}$ or greater target size.

Table 1 and Figure 3 demonstrate an important concept for PV correction. For optimal reconstruction parameters, the resolution and PV loss of a scanner are fixed values characteristic of that scanner, isotope, and reconstruction filters as measured by ACR or NEMA

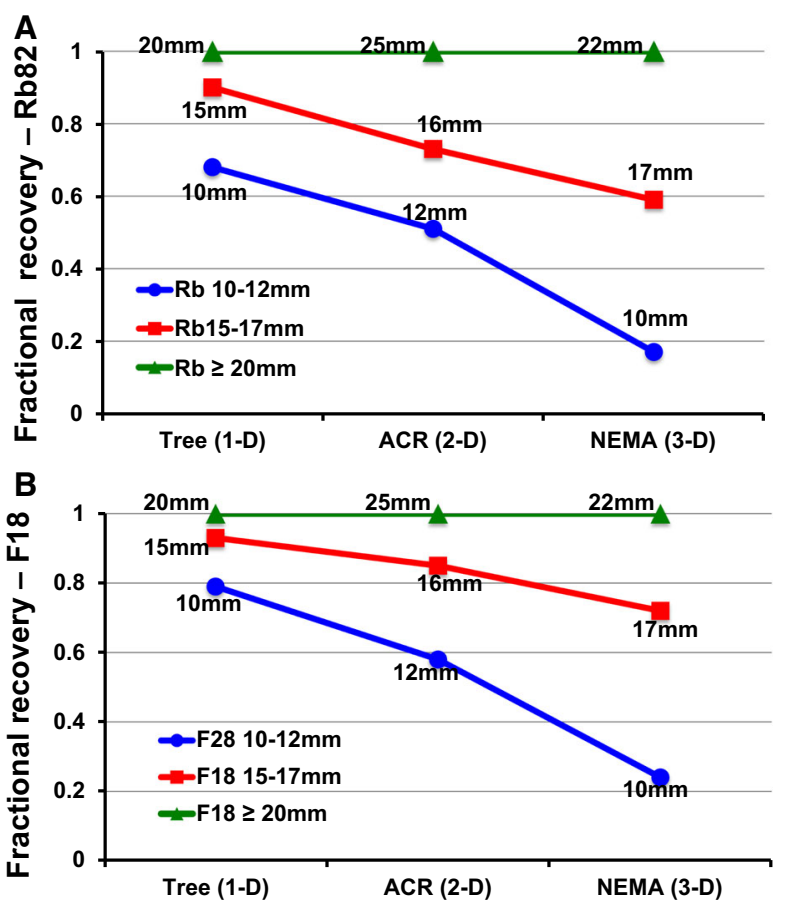

Figure 3. Plots of fractional relative activity recovery for approximately comparable dimensions of three phantoms for $\mathrm{Rb}-82$ (A) and F-18 (B). phantoms. However, for the same reconstruction parameters, Table 1 and Figure 3 also show that for target sizes less than $20 \mathrm{~mm}$, activity recovery and PV loss depend on target size and shape, i.e., whether there are one, two, or three target dimensions. Therefore, PV correction depends on target shape and size separately from and in addition to all the other factors. The aortic and left atrial target sizes for arterial input are usually over $20 \mathrm{~mm}$ and, therefore, do not require PV correction.

In Table 2, PV correction based on PV loss observed for the $16 \mathrm{~mm}$ diameter two-dimensional rods of the ACR phantom overestimates the correction for one-dimensional LV wall thickness. Similarly, PV correction based on PV loss observed for the $17 \mathrm{~mm}$ diameter three-dimensional spheres of the NEMA phantom substantially overestimates correction for one-dimensional LV wall thickness. Both ACR and NEMA phantom data overestimated PV corrections for LV causing erroneously high myocardial perfusion with consequent impaired risk stratification unrelated to validated low flow thresholds of perfusion associated with ischemia, MACE, or death and their reduction after revascularization. ${ }^{7,8}$

Tables 1, 2 and Figure 3 show that the relative activity ratio of two-dimensional ACR $16 \mathrm{~mm} / 25 \mathrm{~mm}$ rods imaged with $\mathrm{Rb}-82$ overestimates the one-dimensional PV correction perfusion incorporating the positron range of $\mathrm{Rb}-82$. However, due to the shorter positron range of $\mathrm{F}-18$, the $\mathrm{PV}$ correction based on the ACR $16 \mathrm{~mm} / 25 \mathrm{~mm}$ rods imaged with F-18 is less than for Rb-82 and largely reduces the overestimated PV correction of the ACR phantom imaged with Rb-82. Therefore, from a practical viewpoint for Rb-82, the PV correction based on the ACR 16/25 mm rods using F-18 approximates the PV correction derived from the onedimensional Tree $15 \mathrm{~mm} / 20 \mathrm{~mm}$ width using Rb-82.

\section{Systematic Impact of Erroneous LV Partial Volume Corrections on Quantitative Perfusion, Risk Stratification, and MACE}

As an example of a patient with CAD undergoing quantitative PET with Rb-82, Figure 4 shows septal and anterior views of abnormal Coronary Flow Capacity (CFC) due to LAD occlusion. For this case, the CFC severity histogram plots and Kolmogorov-Smirnov (KS) statistic compare the cumulative fractional distribution of CFC severity for the entire LV using different PV corrections from Tree, ACR, and NEMA phantoms for quantitative perfusion. For each separate CFC map, its cumulative CFC histogram distribution and its KS statistic uses one-dimensional PV correction derived from the $15 \mathrm{~mm}$ Tree phantom (A) or two-dimensional PV correction derived from the $16 \mathrm{~mm}$ ACR rod (B), or 
Table 2. Rest and stress perfusion, CFR with partial volume corrections based on 1D, 2D, and 3D phantoms ( $\mathrm{N}=186)$ using $\mathrm{Rb}-82$

\section{PET Metric}

Tree 1D stand PVC 0.9

ACR 2D PVC 0.73

NEMA 3D PVC 0.59

Rest $\mathrm{cc} / \mathrm{min} / \mathrm{g}$

Stress $\mathrm{cc} / \mathrm{min} / \mathrm{g}$

CFR

$0.78 \pm 0.07$
$1.35 \pm 0.22$
$1.82 \pm 0.46$

$0.78 \pm 0.07$

$1.82 \pm 0.46$
$1.20 \pm 0.16$
$2.00 \pm 0.42$
$1.78 \pm 0.43$

$1.81 \pm 0.33$

$2.89 \pm 0.76$

$1.70 \pm 0.36$

Paired $\mathrm{T}$ test between all columns for each row $\mathrm{P}<0.000001$

$P V C$, Partial volume correction; CFR, coronary flow reserve

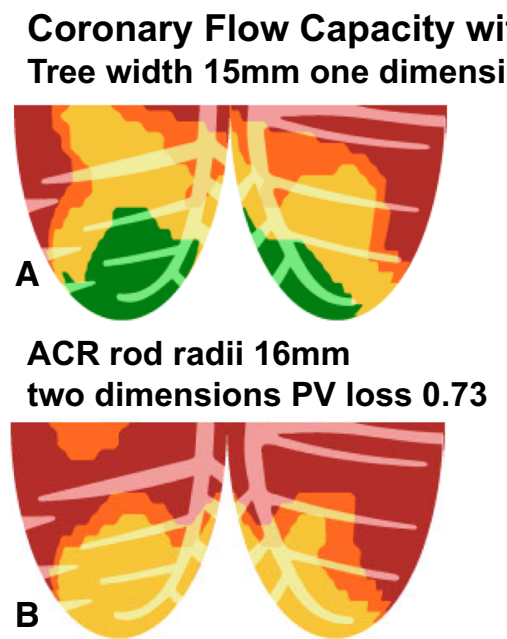

\section{NEMA sphere radii $15 \mathrm{~mm}$ three dimensions PV loss 0.59}

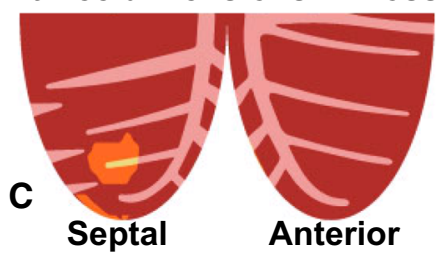

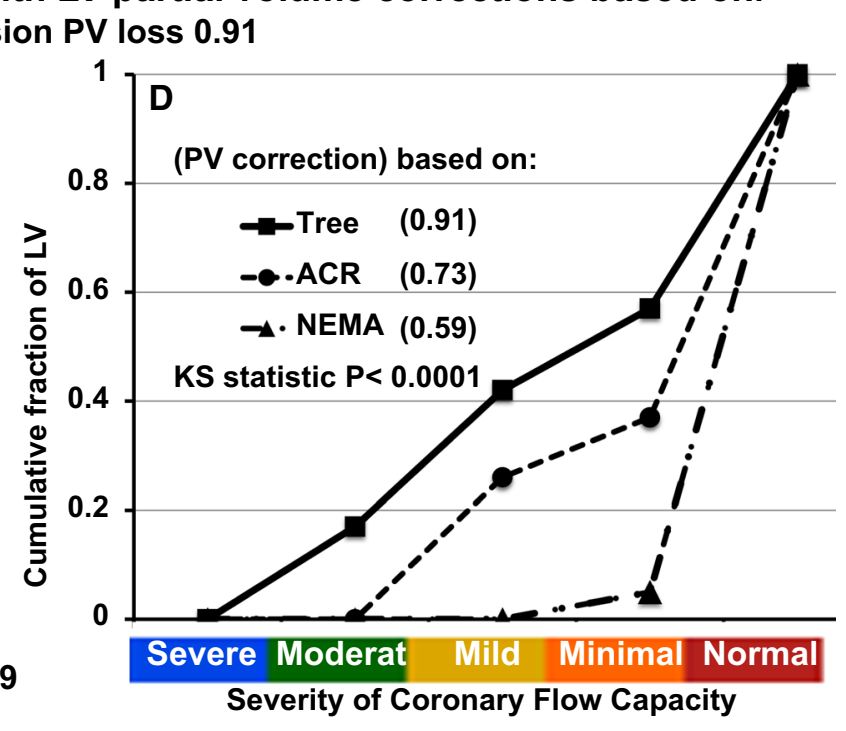

E Global perfusion with PV corrections based on: cc/min/g Tree ACR NEMA Rest $\quad 0.67 \quad 1.05(+57 \%) \quad 1.63(+143 \%)$ Stress $\quad 1.78 \quad 2.63(+48 \%) \quad 3.74(+110 \%)$

Figure 4. Coronary Flow Capacity for one PET case with perfusion and CFC map determined using (A) standard one-dimensional PV correction (tree phantom) compared to using PV corrections derived from ACR (B) and NEMA (C) phantoms with Kolmogorov-Smirnov cumulative histogram plots and statistic (D). PV corrections derived from ACR and NEMA phantomst are expressed as percent increase over the one-dimensional PV correction derived from the tree phantom $(\mathbf{E})$.

three-dimensional PV correction derived from the $17 \mathrm{~mm}$ NEMA sphere $(C)$.

In Figure 4, PV corrections derived from Tree, ACR, and NEMA phantoms progressively increase average global resting and stress perfusion by $50 \%$ to $150 \%$ toward normal or high values that eliminate significant CFC abnormalities despite a known occluded coronary artery. The progressively larger PV corrections derived from Tree to ACR to NEMA phantoms progressively shifts the CFC cumulative severity histogram downward and rightward into erroneously high values within the range of normal PETs.

Figure 5 shows the resulting rest-stress perfusion and CFC cumulative histograms and KolmogorovSmirnov statistic comparing histogram distributions of CFC severities based on the ACR or NEMA phantom 


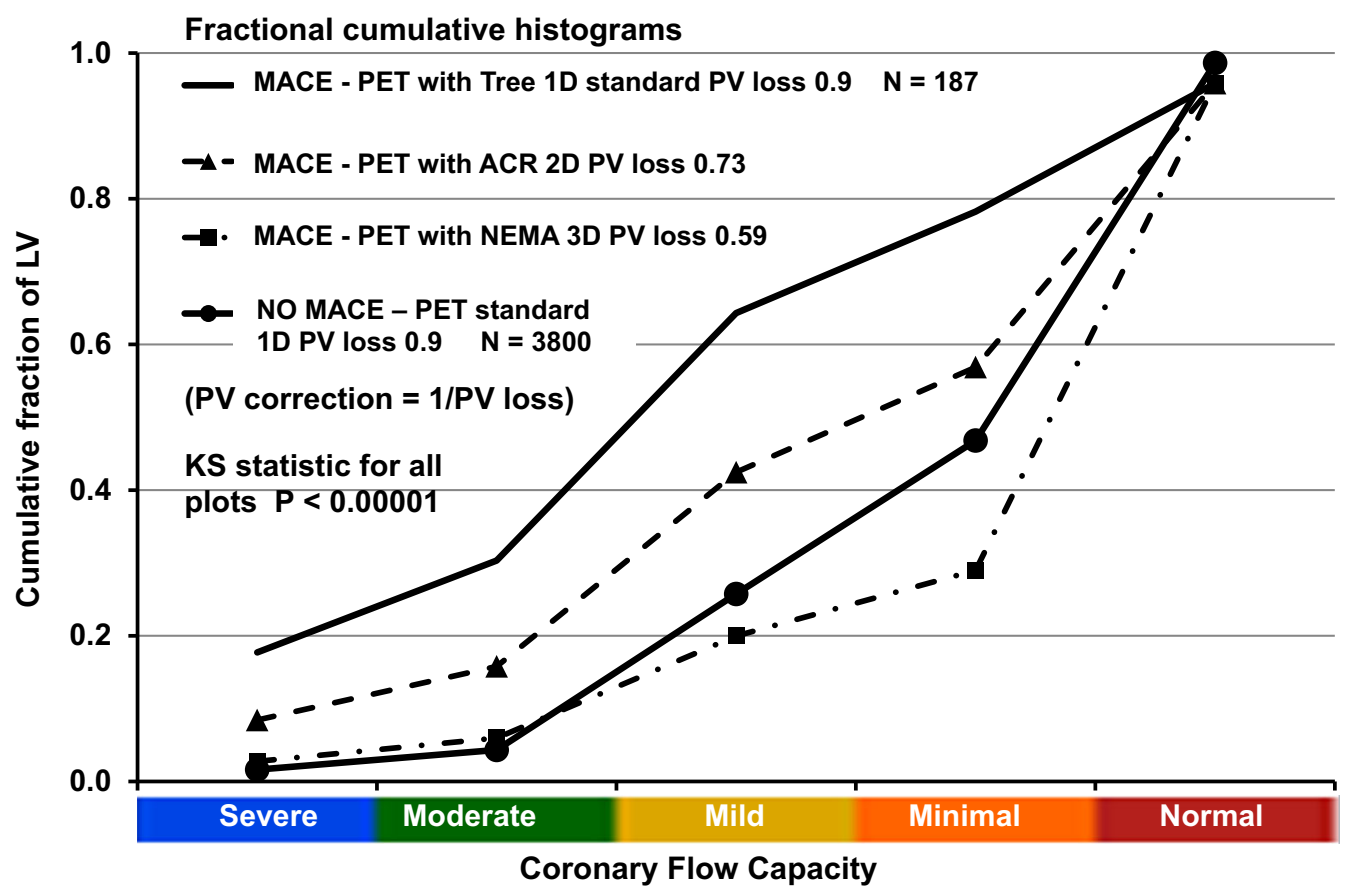

Figure 5. Changes in Coronary Flow Capacity (CFC) due to PV corrections derived from the 1D tree phantom, the 2D ACR rods, and 3D NEMA spheres using MACE over 10-year follow-up as the outcome defined reference of severity for 3987 PET cases without (3800) and with MACE after PET (187). The cumulative histograms and Kolmogorov-Smirnov statistic for CFC of the MACE group based on perfusion using standard one-dimensional PV correction of the tree phantom (solid line) is higher and leftward indicating much worse CFC size-severity compared to the no MACE group (solid line with circles). For larger PV corrections derived from two-dimensional ACR rods (triangles with dashed line) and three-dimensional NEMA spheres (squares with dash dot line), histogram size-severity distribution of PET with MACE shifts downwards and rightwards towards the normal range for PETs with no MACE for PV corrections based on ACR (dashed line with triangles) or NEMA phantoms (dashed-dot line with squares line with triangles) that are uncorrected to 1D PV by our simple formula. For the NO MACE group with PV corrections based on 2D ACR or 3D NEMA PV loss, the CFC size-severity histogram distributions as \% of LV are also shifted downward and rightward toward larger \% of LV in the high flow red (normal) or orange (minimally reduced) range and hence of no clinical consequence compared to the $1 \mathrm{D}$ PV corrections for the MACE group, hence not shown.

PV corrections for the PETs without and with subsequent MACE.

The progressively larger PV corrections derived from Tree to ACR to NEMA phantoms progressively shifts the CFC cumulative severity histogram downward and rightward into erroneously high values toward or within normal ranges. The resulting high perfusion levels are well above the CFC threshold associated with clinical ischemia (4-16) and high risk of death or $\mathrm{MI}^{6,7}$ that are reduced by revascularization. ${ }^{7}$ Accordingly, the overestimated PV corrections and overestimated perfusion values derived from ACR and NEMA phantoms may impair accurate risk stratification and hence potential coronary interventions guided by perfusion severity.

\section{Validation of PV Correction Equation from ACR and NEMA Phantoms}

As demonstrated in Table $1, R$ is the activity recovery loss due to $\mathrm{Rb}-82$ positron range relative to $\mathrm{F}-18$ that is 0.96 for $\mathrm{Rb}-82(0.90 / 0.94$ in Table 1$)$ and 1.0 for F-18.

For an observed relative activity recovery of 0.85 from the 16/25mm ACR rods, the PV correction for the one-dimensional PV correction for LV imaging with F18 is calculated as

PVD1 $=(0.85 / 1)^{1 / 2}$ or $^{2} \sqrt{ } 0.85=0.92$ that compares with the directly measured activity recovery of 0.94 for F-18 from the $15 \mathrm{~mm} / 20 \mathrm{~mm}$ activity ratio of the Tree width in Table 1. 
For cardiac Rb-82 imaging, PV activity recovery for the same observed activity recovery of F-18 in the ACR and NEMA phantoms would be as follows. For an observed activity recovery of 0.73 from the $16 \mathrm{~mm} /$ $25 \mathrm{~mm}$ ACR spheres, the PV correction for the onedimensional PV correction for cardiac imaging with $\mathrm{Rb}$ is calculated as: PVD1 $=\left(0.73 / 0.96^{2}\right)^{1 / 2}$ or $\sqrt{0} 0.79=$ 0.89 that compares with the directly measured activity recovery of 0.9 for $\mathrm{Rb}$ from the $15 \mathrm{~mm} / 20 \mathrm{~mm}$ relative activity ratio of the Tree width in Table 1 . The Online Resource-1 has additional examples of this equation for the NEMA phantom.

\section{Advanced, High-Resolution 3D PET-CT Scanners-Progressive Partial Volume Loss with Limited Size of 1 Dimension (Tree Phantom), 2 Dimensions (ACR), and 3 Dimensions (NEMA)}

Figure 6 shows PV corrections for F-18 in the ACR phantom for three different advanced, high-resolution, 2D (GE DSTE)(A) and 3D PET-CT scanners, GE D710
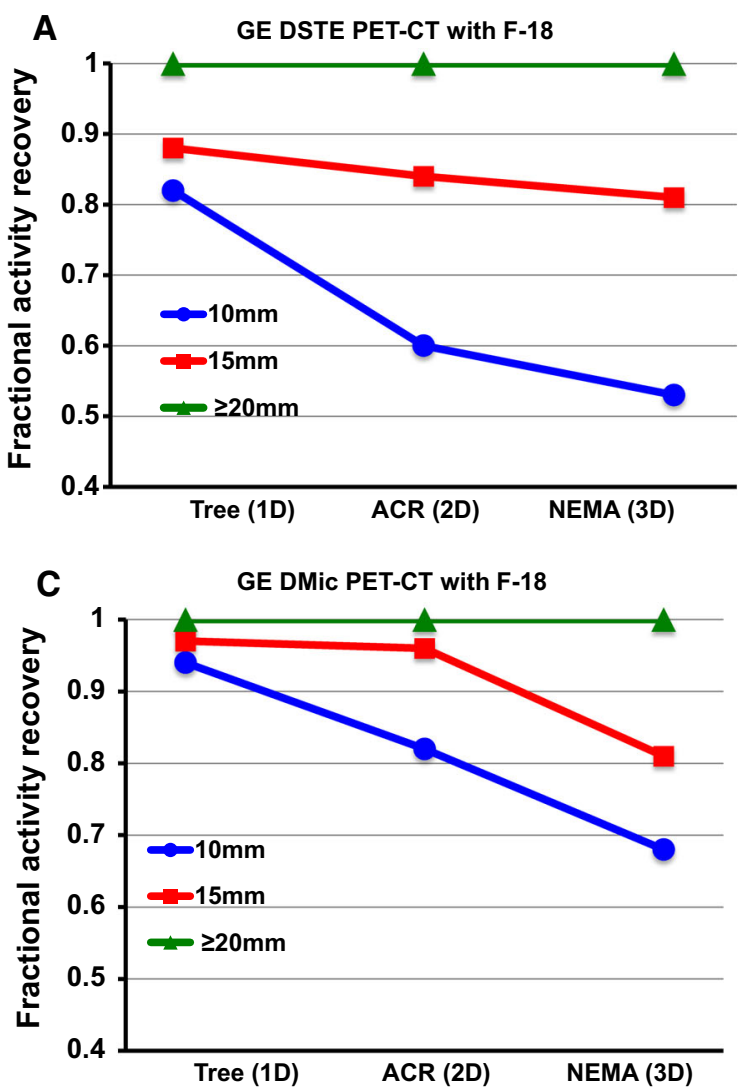

(B), and DMic (C). For comparison among these scanners (D), relative activity recovery from different sized phantom targets was extrapolated to match the $10 \mathrm{~mm}, 15 \mathrm{~mm}$, and $20 \mathrm{~mm}$ sizes of the tree phantom for simplified correct comparative plots in Figure 6D. All scanners show the same trend as the older Discovery ST PET with 16-slice CT with decreasing activity recovery for the same size targets of the one-dimensional Tree phantom (branch width) to two-dimensional ACR rods (two equal radii) to three-dimensional NEMA spheres (three equal radii). This observation suggests a general principle for cardiac PET-CT scanners for the one-dimensional PV loss related to LV wall thickness where the partial volume correction value is specific and needs determining for each different type of scanner using one of these phantoms.

\section{DISCUSSION}

For quantitative activity recovery by PET, our phantom data confirm a basic principle that PV loss of peak activity progressively declines with each added
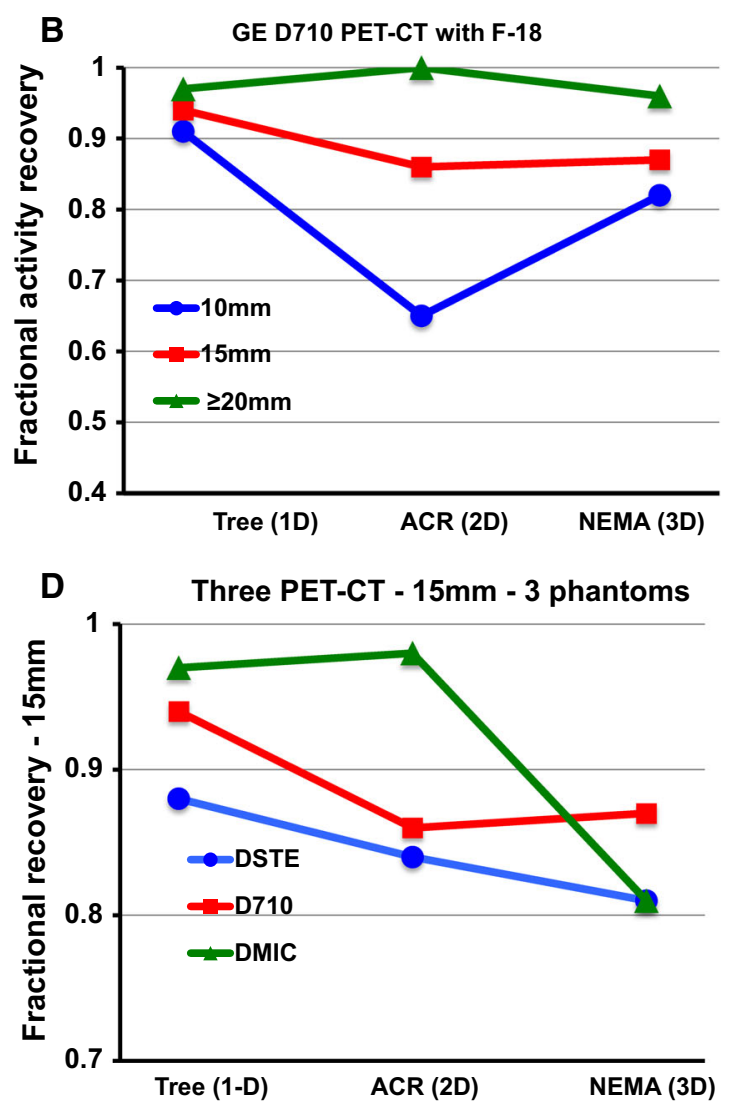

Fig. 6. Fractional relative activity recovery for GE DSTE (A), D710 (B), DMic (C), and for three 3D PET-CT scanners (D) for $10 \mathrm{~mm}, 15 \mathrm{~mm}$, and $\geq 20 \mathrm{~mm}$ of one-dimensional tree phantom, twodimensional ACR rods, and three-dimensional NEMA spheres filled with F-18. 
target dimension smaller than $20 \mathrm{~mm}$. Consequently, quantifying myocardial perfusion requires a one-dimensional PV correction for the single dimension of $\mathrm{LV}$ wall thickness since circumferential and longitudinal dimensions are greater than scanner resolution with no significant PV loss in those dimensions. Since ACR rods have two dimensions (two equal radii) and NEMA spheres have three dimensions (three equal radii) less than $20 \mathrm{~mm}$, partial volume corrections based on these phantoms need to be corrected to the equivalent of a one-dimensional target by an equation as proposed and validated in this manuscript. As a simple practical step, the optimal LV myocardial PV loss and correction for Rb-82 and F-18 can be derived from the widely available ACR phantom filled with F-18 as the relative activity ratio of the $16 \mathrm{~mm} / 25 \mathrm{~mm}$ diameter rods.

\section{Limitations}

PV loss and corrections vary with systolic-diastolic LV wall thickness and hence with heart rate since tachycardia during pharmacologic stress systole increases cumulative systolic time. However, timevarying PV corrections during diastole and systole proved complexly impractical ${ }^{4}$ and substantially less important than the correct, fixed, one-dimensional PV correction for an approximate fixed LV wall thickness of $15 \mathrm{~mm}$.

\section{COMPARISON TO THE LITERATURE}

Commonly, PV corrections may be determined by compartmental flow modeling with PV correction as one of several unknowns whose value is solved by curve fitting to time activity curves. ${ }^{1,2}$ This method assumes very high fidelity, correct, time activity curves within a fixed ROI on the LV. However, the values of these PV corrections are not listed or published but "buried", within the flow model equations. ${ }^{1,2}$ Consequently, this model is less suited for studying effects of variable partial volume corrections on perfusion values defining regional physiologic $\mathrm{CAD}$ severity to guide management or related to MACE.

Moreover, time activity curves from this sample volume may be corrupted by cardiac motion, translation, and wall thickening within a varying attenuation environment as the heart commonly moves up to $20 \mathrm{~mm}$ vertically and medially superimposed on respiratory positional changes of the heart, mediastinum and surrounding attenuation structures. ${ }^{4,5,8-10}$ In addition, as the sample volume for time activity curves decreases in size from an entire arterial distribution down to secondary branches, to one $\mathrm{cm}^{3}$ or one pixel, the time activity curves are increasingly subject to random count variability (statistical noise) thereby limiting reliability of regional time activity curves for regional quantitative perfusion of actual arterial distributions.

\section{NEW KNOWLEDGE GAINED}

For quantifying myocardial perfusion by PET to predict high-risk CAD that is significantly reduced by revascularization, partial volume loss due to $\mathrm{LV}$ wall thickness less than scanner resolution can be corrected by cardiac specific adaptation of phantoms with limited size in 1 dimension (Tree phantom), 2 dimensions (ACR), and 3 dimensions (NEMA) for established 2D or advanced, high-resolution 3D PET-CT scanners that has not been previously demonstrated.

\section{CONCLUSIONS}

Since the LV circumferential and longitudinal dimensions are large compared to scanner resolution, myocardial partial volume loss is due to the single dimension of $\mathrm{LV}$ wall thickness averaging $15 \mathrm{~mm}$ for diastole and systole, hence needing one-dimensional partial volume correction. Partial volume activity loss and corrections based on 2 dimensional ACR rods and 3 dimensional NEMA spheres overestimate PV correction for both $\mathrm{Rb}-82$ and F-18 with corresponding erroneously high-quantitative perfusion by $+50 \%$ to $+150 \%$ compared to reference values validated clinically by outcomes data. $^{7,8}$

Therefore, accurate PV loss and its correction are optimally derived from a one-dimensional phantom imaged with $\mathrm{Rb}-82$ or F-18 to obtain the relative ratio of the $15 \mathrm{~mm} / 20 \mathrm{~mm}$ wide single dimension target. Since ACR and NEMA phantoms are widely available, their overestimated PV loss and corrections for quantitative LV myocardial perfusion can be corrected to the needed one-dimensional partial volume correction with a simple equation applicable to all cardiac PET-CT scanners.

\section{Disclosure}

None of the authors have any conflicts of interest to disclose.

\section{Open Access}

This article is licensed under a Creative Commons Attribution 4.0 International License, which permits use, sharing, adaptation, distribution and reproduction in any medium or format, as long as you give appropriate credit to the original author(s) and the source, provide a link to the Creative Commons licence, and indicate if changes were made. The images or other third party material in this article are included in the article's Creative Commons licence, unless 
indicated otherwise in a credit line to the material. If material is not included in the article's Creative Commons licence and your intended use is not permitted by statutory regulation or exceeds the permitted use, you will need to obtain permission directly from the copyright holder. To view a copy of this licence, visit http://creativecommons.org/licenses/by/4.0/.

\section{References}

1. Klein R, Beanlands RSB, deKemp RA. Quantification of myocardial blood flow and flow reserve: Technical aspects. J Nucl Cardiol 2010;17:555-70.

2. Renaud JM, DaSilva JN, Beanlands RS, DeKemp RA. Characterizing the normal range of myocardial blood flow with ${ }^{\mathbf{8 2}}$ rubidium and ${ }^{\mathbf{1 3}} \mathrm{N}$-ammonia PET imaging. J Nucl Cardiol 2013;20:578-91.

3. Yoshida K, Mullani, N, Gould KL. Coronary flow and flow reserve by positron emission tomography simplified for clinical application using Rb-82 or N-13 ammonia. J Nuc Med 1996;37:17011712.

4. Johnson NP, Gould KL. Partial volume correction incorporating $\mathrm{Rb}-82$ positron range for quantitative myocardial perfusion PET based on systolic-diastolic activity ratios and phantom measurements. J Nucl Cardiol 2011;18:247-58.

5. Kitkungvan D, Johnson NP, Roby AE, Patel MB, Kirkeeide R, Gould KL. Routine clinical quantitative rest stress myocardial perfusion for managing coronary artery disease: Clinical relevance of test-retest variability. J Am Coll Cardiol CV Imaging 2017;2017(10):565-77.
6. Gould KL, Johnson NP. Coronary physiology: Beyond CFR in microvascular angina. J Am Coll Cardiol 2018;72:2642-62.

7. Gould KL, Johnson NP, Roby AE, Nguyen T, Kirkeeide R, Haynie $\mathrm{M}$, et al. Regional artery specific thresholds of quantitative myocardial perfusion by PET associated with reduced MI and death after revascularization in stable CAD. J Nucl Med 2019;60:410-7.

8. Gould KL, Pan T, Loghin C, Johnson N, Guha A, Sdringola S, et al. Frequent diagnostic errors in cardiac PET-CT due to misregistration of CT attenuation and emission PET images: A definitive analysis of causes, consequences and corrections. J Nucl Med 2007;48:1112-21.

9. Gould KL, Pan T, Loghin C, Johnson NP. Sdringola. reducing radiation dose in rest stress cardiac PET-CT by single post stress cine CT for attenuation correction-Quantitative validation. J Nucl Med 2008;49:738-45.

10. Vasquez AF, Johnson NP, Gould KL. Variation in quantitative myocardial perfusion due to arterial input selection. J Am Card Cardiol CV Imaging 2013;6:559-68.

11. Bachrach SI, Carson RE. In hot blood. J Am Coll Cardiol CV Imaging 2013;6:569-73.

12. Johnson NP, Gould KL. Integrating noninvasive absolute flow, coronary flow reserve, and ischemic thresholds into a comprehensive map of physiologic severity. J Am Coll Cardiol: Cardiovasc Imaging 2012;5:430-40.

13. Johnson NP, Gould KL. Physiologic basis for angina and ST change: PET-verified thresholds of quantitative stress myocardial perfusion and coronary flow reserve. J Am Coll Cardiol 2011;4:990-8

Publisher's Note Springer Nature remains neutral with regard to jurisdictional claims in published maps and institutional affiliations. 